DOI: 10.2478/romneu-2013-0013

\title{
Traumatic intracranial aneurysms
}

\author{
A. Chiriac, B. Iliescu' ${ }^{1}$, Z. Faiyad ${ }^{1}$, I. Poeata \\ "Grigore T. Popa" University of Medicine and Pharmacy, Iasi \\ 1"Prof. Dr. N. Oblu” Clinic Emergency Hospital, Iasi
}

\begin{abstract}
Traumatic intracranial aneurysms are rare conditions that can be a result of vast head trauma. Different aspects as history, etiology, histopathology, clinical manifestations, diagnosis and treatment options are discussed regarding the literature. A particular case of a pericalosal aneurysm is presented to highlight the importance of keeping in mind that this location can be a site of such aneurysms.
\end{abstract}

\section{Introduction}

Traumatic intracranial aneurysms are rare conditions and represents less than $1 \%$ of all intracranial aneurysms. (1) They can be a result of a penetrating or nonpenetrating head trauma and are most frequently located in the peripheral cerebral vasculature or close to the skull base. Because traumatic aneurysms are fragile vascular lesions with high risks of rupture, early diagnosis and prompt treatment are essential.

\section{Historical notes (2)}

In 1829, Smith reported for the first time an autopsy-proven case of a middle meningeal artery aneurysm after head injury. Later, in 1891, Bollinger postulated that some instances of "delayed apoplexy" after head injury were due to the rupture of a traumatic aneurysm. Although Guibert in 1985 reported a case involving the infraclinoid internal carotid artery, Birley and Trotter in 1928 were the first who describe a case of an intracranial aneurysm after severe head injury. They were followed by Tonnis and Cairns in 1934 and 1942 who demonstrated the first case proven by angiography and provided a comprehensive description of these aneurysms. In 1949 Krauland described an aneurysm formation after a depressed skull fracture and angiographically evidence of these lesions after a closed head injury were demonstrated in 1962 (Hirsch et al 1962; Burton et al 1968).

Beginning with 1970s the frequency of their diagnosis has decreased due to the application of computerized tomography (rather than traditional angiography) as the modality of imagistic evaluation of headinjured patients. Conventional cerebral angiography remains the reference standard for the diagnostic work-up of intracranial aneurysms (Dubey et al 2008).

\section{Etiology}

Traumatic intracranial aneurysms may result from vast but commonly causes as sports accidents, assault or traffic accidents (pedestrians or in vehicle passengers. They have been described in association with both blunt and penetrating trauma, with the former being more commonly reported. (5)

Several mechanisms have been found responsible for traumatic aneurysms formation. All involve either direct injury to the vessel or stretching of the vessel by adjacent forces. The mechanism of injury is closely related to the anatomical location of the involved artery. Infraclinoid carotid and 
basilar artery aneurysms are commonly associated with basilar skull fractures, which are explained by the close relation of these vessels with the skull base. In the supraclinoid segment the carotid artery is characterized by a transitions zone, from a relatively fixed structure in the skull base and cavernous sinus to a relatively mobile structure as it ascends in the cisternal spaces. There are hypothesis that, either movement of the supraclinoid segment against the anterior clinoid process or stretching of the carotid artery at this transition zone may leads to an aneurysm formation. (5)

Concerning the distal subcortical aneurysms occurrences there are predominantly along the anterior cerebral artery and its branches. The close relation of these vessels along much of their length to the falx cerebri has led some authors to the hypothesis that traumatic movement of the brain and vessels against the relatively fixed falx cerebri can lead to aneurysm formation. (5) In the same siuation, posterior cerebral artery aneurysms are thought to be the result of trauma of the vessel against the tentorium. (5) Also, the distal cortical aneurysms may occur in association with linear or depressed skull fractures and dural lacerations, commonly involving the middle cerebral artery or ACA. (5) It is believed that temporary or prolonged herniation of the cortical vessel up into the fracture defect leads to direct injury to the vessel wall. (5)

Ventureyra and Higgins proposed 4 categories of the mechanism of causation of traumatic intracranial aneurysms, as closed head injuries, missile injuries, penetrating head injuries, and iatrogenic injury. In their review of 130 traumatic intracranial aneurysms in children, closed head injuries was found to constitute $72 \%$ of cases, missile injuries $16 \%$, iatrogenic injury $8 \%$, and penetrating head injury $4 \%$ of cases. Minor closed head injuries without fracture or significant initial impairment of consciousness can also produce traumatic intracranial aneurysms. Traumatic intracranial aneurysms due to closed head injuries can result from rapid deceleration causing sudden brain and artery movement producing vessel wall injury against stationary structures. (4)

Developments of iatrogenic traumatic aneurysms have been described by some authors after a variety of procedures, including endoscopic ventriculostomy, intranasal procedures, intracranial surgery, and repeated subdural taps.

\section{Histopathology}

From histological point of view, traumatic aneurysms can be classified as true, false or mixed aneurysms. The classification is based on both the anatomy and mechanism of traumatic aneurysm formation. True aneurysms are characterized by incomplete mural injury of the intima and variable involvement of the internal elastic layer and media that produces a localized weakening of the vessel wall and aneurysm formation. False aneurysms are considered to be the most common histological type that results from disruption of all three layers of the vessel wall with formation of a contained hematoma outside the vessel. A false lumen formation then develops, creating an aneurysmal dilation. Because the adventitia of the native vessel usually is intact, the phenomenon is being presumably secondary to flow dynamics against the weakened vessel wall. These are presumably the histological type associated with penetrating injuries. The third histological types, the mixed aneurysms, are initially true aneurysms that subsequently undergo a contained rupture forming hematoma and false lumen. The term of 
"mixed aneurysm" is used by some authors to describe saccular aneurysms that occur in association with dissection of the parent. (4, 5)

However, because the most case reports contain little or no histological data, the relative incidence of these histological types is not well known. The histological classification is of little benefit in terms of clinical management because intervention is required regardless of the type or mechanism.

Ventureyra and Higgins proposed a classification of paediatric traumatic intracranial aneurysms, into early onset (occurring within 4 weeks after injury) or late onset (occurring beyond 4 weeks). Most reported cases of traumatic intracranial aneurysms presented with early onset. Late onset manifestation in paediatric traumatic intracranial aneurysms, as in the present case is rare with only about 10 reported cases in literature till date. They often result from $\mathrm{CHI}$ and manifest clinically as aneurysmal masses. (5)

\section{Location}

The location of traumatic intracranial aneurysms varies, but these are commonly found in the anterior circulation. The majority of traumatic intracranial aneurysms occur in the supraclinoid segment of the carotid artery and along the anterior cerebral artery and its branches, particularly the pericallosal and callosomarginal arteries. However, reported locations are the vertebral artery, internal carotid artery, anterior cerebral artery, middle cerebral artery and middle meningeal artery.

Asari at al observed that the most common location for traumatic aneurysms was the middle cerebral artery, followed by the distal anterior cerebral artery. Traumatic aneurysms of the skull base usually involve the petrous, cavernous and supraclinoid ICA. Petrous and cavernous ICA traumatic aneurysms are usually associated with basilar skull fractures. Supraclinoid ICA aneurysm may form as a result of blunt arterial contusion by the anterior clinoid process or sudden stretching of the artery during impact and/or they may be associated with orbital roof or anterior clinoid process fractures. (3)

\section{Clinical manifestations}

The clinical manifestations of traumatic intracranial aneurysms are usually similar with that of normal aneurysms. However, direct neural injuries and associated impairment of consciousness produced by head injury often mask the early symptoms due to traumatic intracranial aneurysms, leading to delayed diagnosis.

Typically, traumatic intracranial aneurysms range in size from 2 to $15 \mathrm{~mm}$ (Salar et al 1978; Achram et al 1980; (Haddad et al 1991). Most present 2 to 8 weeks after injury, but some present earlier and others are delayed by months or years (Fleischer et al 1975; Aarabi 1988; Haddad et al 1991; Ventureyra and Higgins 1994). $(3,5)$

The most common symptoms include an acutely decreased level of consciousness, seizure, or focal neurological deficit. In a typical patient, the level of consciousness is initially depressed due to the trauma itself followed by a lucent period after which secondary deterioration occurs because of intracranial hemorrhage (Sasaoka et al 1997) (2).

Imaging scanning usually demonstrates acute intracranial hemorrhage, which may be subarachnoid, intraparenchymal, intraventricular, or subdural depending on aneurysm location. (5) The average time from initial trauma to traumatic aneurysmal hemorrhage is approximately 21 days and is associated with a mortality rate as high as 
$50 \%$. (5) Patients with infraclinoid carotid artery aneurysms can present with cranial nerve deficits, diabetes insipidus, recurrent or massive epistaxis, unilateral blindness, or symptoms of a cavernous-carotid fistula. (5) A later compressive cranial nerve palsies may occur due to enlarging aneurysm, but an abducens palsy can be present within the first few days because of raised intracranial pressure (2). The epistaxis appears when a petrous or intracavernous internal carotid artery aneurysm is associated with a basal skull fracture ruptures. Patients with supraclinoid carotid artery lesions can present with headache, memory disturbance, and progressive visual loss prior to rupture; such symptoms have been reported to occur for as long as 7 years prior to diagnosis. (5) Some cases have presented with hydrocephalus, involving pericallosal aneurysms and with a distal cortical lesion.

Distal cortical aneurysms have unique characteristics that may make them more likely to be diagnosed prior to the occurence of hemorrhage. These aneurysms can lead to the development of a growing skull fracture that becomes physically palpable months to years after the injury. (5) Buckingham and colleagues found 11 reported cases of distal cortical aneurysms associated with blunt trauma; seven of these patients (63. 6\%) presented without hemorrhage and were diagnosed primarily with either on routine radiographic follow up or by evaluation of growing skull fractures. However, only 20. $5 \%$ (of 44) of blunt traumatic aneurysms were diagnosed prior to hemorrhage in more proximal locations. $(2,5)$

The otic hemorrhage is rare condition. In infants, a skull fracture may "grow" concomitant with the enlargement of an aneurysm because the skull sutures are not fused (Endo et al 1980). The aneurysm may be asymptomatic and discovered incidentally in $10 \%$ to $20 \%$ of cases during neuroimaging. A recent case repot discloses acute sanguinous ventriculostomy output, suggesting underlying aneurysm rupture (Dubey et al 2008). (2)

\section{Diagnosis}

In the early part of the century, clinicians did not have access to the neuroimaging modalities such as CT and MR imaging. The direct diagnosis of a traumatic aneurysm could only be made on the angiography, in the operating room, or at autopsy (5). The diagnosis of traumatic intracranial aneurysms requires a high index of suspicion (3). Suspicion should be made in all head injured or post-operative patients with delayed neurological deterioration, delayed haemorrhage, unexplained major arterial bleeding during evacuation of the haematoma, new or unexplained intracranial bleed, discrepancy between the amount of subarachnoid haemorrhage, intraventricular haemorrhage, facio-orbito-pterional injuries, penetrating fragments, especially if they cross the midline or traverse into another dural compartment. (5)

Possibility of emergency CT scanning has allowed clinicians to diagnose delayed intracranial hemorrhage due to traumatic aneurysmal rupture more rapidly and more frequently than previously possible. For patients suffering closed head injury immediate CT scanning should be undertaken as soon as possible. Thus, cerebral angiography should be considered in patients with delayed neurological deterioration or with CT scan evidence of vascular injury. Cerebral angiography is instrumental in the diagnosis of traumatic aneurysms and also helpful in differentiating them from congenital aneurysm. Imagistic features on angiography such as absence or poorly defined neck, unusual sites (peripheral 
location other than at a branching point) or projections of the aneurysm, irregularly contoured aneurysmal sac, and delayed filling and emptying of the aneurysm could suggest a traumatic etiology. $(4,5)$

Magnetic resonance angiography (MRA) with time of flight (TOF) MRA alone or combined with standard spin-echo imaging should be considered in the diagnosis of spontaneous intracranial aneurysms especially in patients with history of trauma and recurrent epistaxis, visual loss, progressive cranial nerve palsy or an enlarging skull fracture. Recently, Ida et al (18) reported a $90 \%$ true-positive diagnostic rate in detection of traumatic intracranial aneurysm. However, so far no studies on efficacy of MRI/MRA in diagnosis of have been reported. $(3,4)$

More recent literature describe several cases in which patients with normal angiography subsequently present with delayed hemorrhage and have aneurysms that are then revealed on repeated studies. Also, some authors of several large series recommend routine angiography 2 weeks after the injury to rule out the delayed aneurysm formation especially for penetrating injuries and particularly stab wounds.

Today, an earlier definitive diagnosis of traumatic intracranial aneurysm seems to be associated with a trend toward more aggressive surgical treatment, and better outcomes than in earlier cases.

\section{Treatment options}

Once diagnosed, traumatic intracranial aneurysms required an emergency management because they rarely regress and have a high incidence of rupture (as high as $67 \%$ in some studies). (3) The management is mainly directed to prevent recurrent hemorrhage and surgical repair and endovascular interventions. Usually, the treatment approach is carried out on a case to case basis since atypical locations and variable anatomical features are often encountered. Surgical techniques option includes aneurysmal clipping, aneurysm resection with or without arterial bypass, or trapping of the aneurysm. Such treatment requires careful planning and evaluation such as preoperative test occlusion of the vessel in preparation for a trapping procedure. In case of impossibility clip obliteration of the aneurysm it may need to be trapped with an accompanying vascular bypass. Thus, the surgical approach should allow easy acces to the superficial temporal artery or the saphenous vein for bypass grafting and also, a proximal and distal control. When aneurysm trapping or clipping is not possible wrapping with piece of muscle secured with clips may be employed. (3)

The endovascular option should be considered in the difficult aneurysms. Endovascular interventions may include embolization with liquid agents, detachable balloons, or coils which may require assistance with balloon or a stent placement. There are also situations when patient's clinical condition may also limit treatment options. Unstable patients that not be suitable to undergo general anesthesia, patients with active bleeding that cannot be treated by dual antiplatelet therapy for stent assisted aneurysm coil embolization are special cases that need combined treatment.

Fleischer and coworkers reported in 1975 a 41\% mortality rate for patients with conservative treatment and a $18 \%$ mortality rate in surgically treated patients. If the surgical clipping has the advantages of definitive aneurysm occlusion, vascular reconstruction with parent artery preservation or mass effect removable, the endovascular techniques avoids prolonged anesthesia, minimizes manipulation of 
adjacent vessels and structures, and allows immediate angiography control. (5)

\section{Case presentation}

A 72-year-old woman slipped on ice and fell down from a steers one month prior to admission in this hospital. $\mathrm{He}$ was asymptomatic, except for headache and dizziness for 2 days. He was treated by a neurologist in a local hospital and was investigated by cranio-cerebral computed tomography with enhancement. Imaging CT demonstrates no brain hemorrhage or vascular lesion (Figure 1).

After 1 month, she developed sudden onset of headache and vomiting in time of slipping followed by confusion syndrome. She was readmitted for the same and later referred to this hospital for further treatment.

MRI of brain (Figure 2) revealed well defined lobulated hypo-intense lesion on T2-TRIM-dark-fluid and T2-TSE sequences in the region of distal anterior cerebral artery. She improved gradually over next 7 days and was sent to our hospital for further treatment. At admission, she was conscious with a GCS of 14. A cerebral CT-angiography (Figure 3 ) was performed and revealed a left sided anterior cerebral artery aneurysm just proximal to its bifurcation into callosomarginal and pericallosal arteries. A repeat native CT scan of brain revealed no hemorrhage or hydrocephalus.

On the same day day, left frontal craniotomy and clipping of the aneurysm by inter-hemispheric approach was performed.

By microdissection and concomitant retraction of the medial surface of the left frontal lobe, the pericallosal arteries were identified distally. They were followed towards to suspected site of aneurysm and proximal portion of the right pericallosal artery was identified.

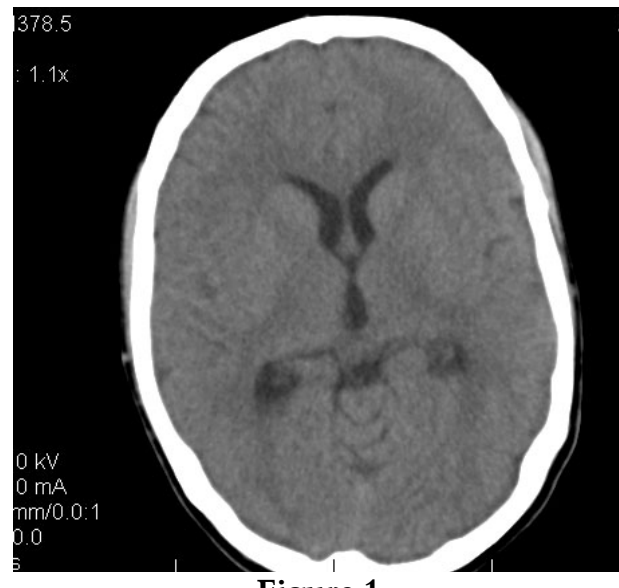

Figure 1

Initial CT scan (after head trauma)
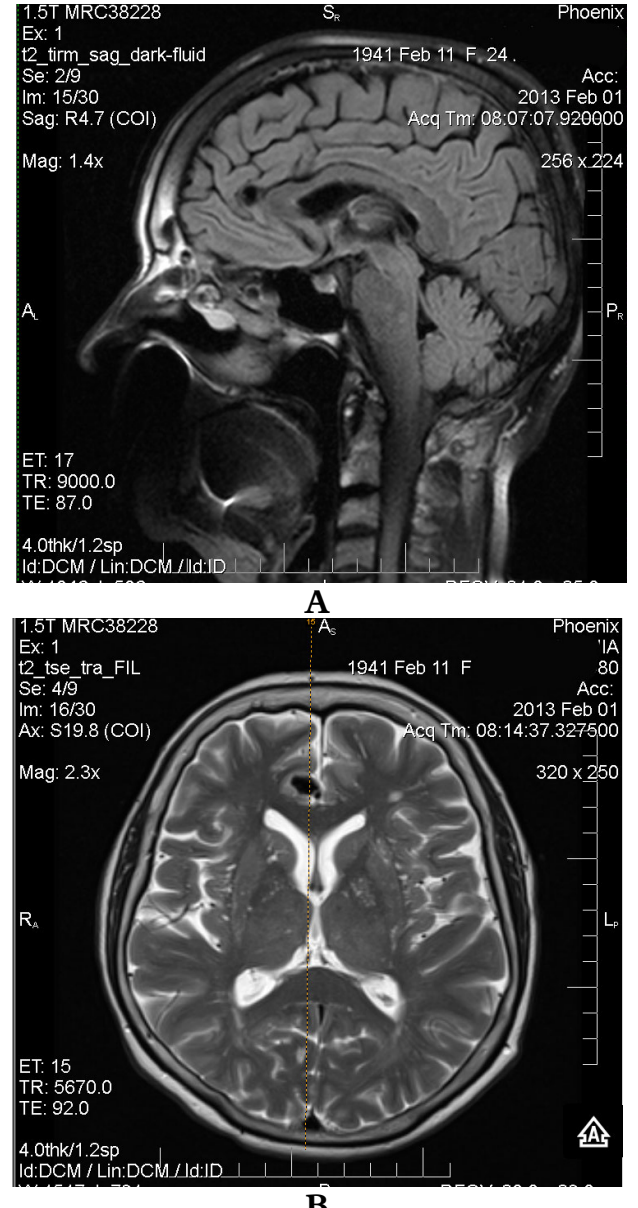

B

Figure 2A, B

T2-TRIM-dark-fluid and T2-TSE sequences showing a vascular lesion in the region of distal anterior cerebral artery 
DOI: 10.2478/romneu-2013-0013

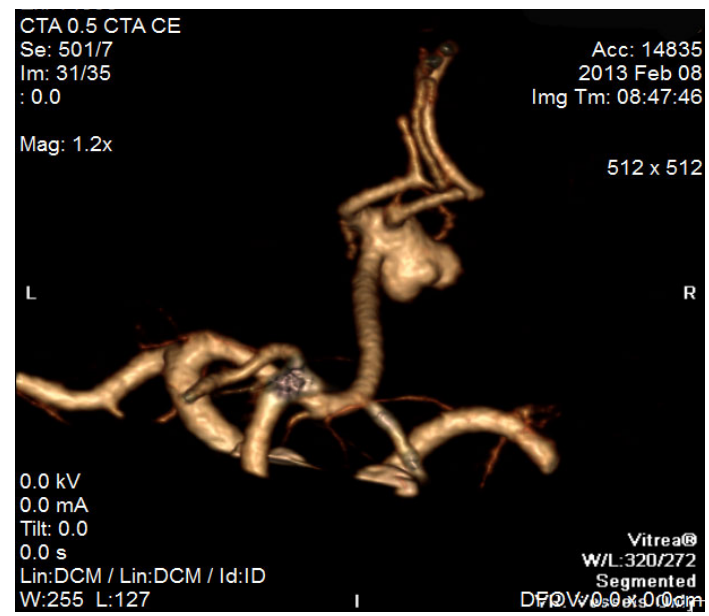

Figure 3

3D CT angio reconstruction showing a a left sided anterior cerebral artery aneurysm

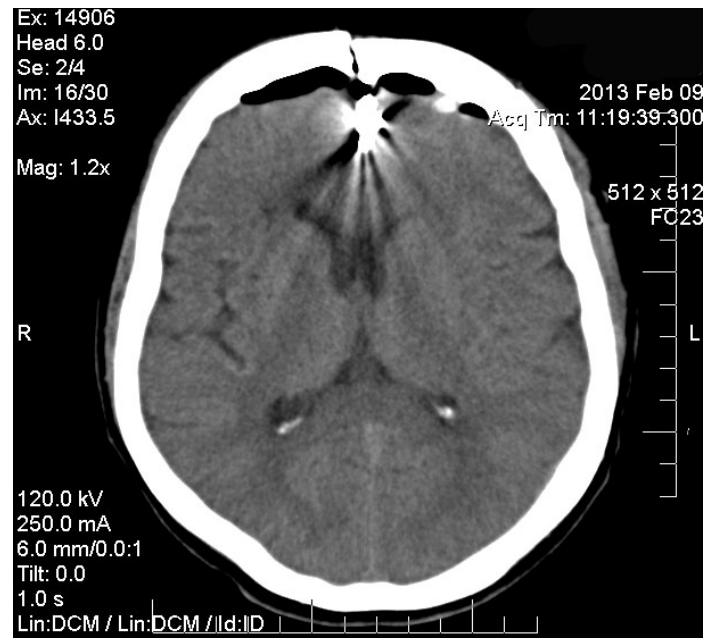

Figure 4

Postoperative CT scan
After having achieved proximal control, the aneurysm was dissected and the neck reconstructed with three yasargil clips for aneurysm complete occlusion. Post operative period was uneventful and a CT scan was performed before discharge 7 days later (Figure 3).

\section{Conclusions}

Traumatic intracranial aneurysms are rare, but must be considered in patients with acute neurological deterioration after closed head injury. Usually they occur more commonly in children than adults. The appropriate management of these lesions may include surgical clipping, excision or trapping procedure and/or endovascular occlusion.

\section{References}

1. Jefferson T Miley, Gustavo J Rodriguez, Adnan I Qureshi: Traumatic Intracranial Aneurysm Formation following Closed Head Injury, Journal of Vascular and Interventional Neurology 2008; 1(3):79-82;

2. Zachary H Dodd, Mitesh V Shah, Steven R Levine: Traumatic intracranial aneurysms, MedLink Neurology 2013;

3. Deepak Awasthi, Greg Dowd: Lessons Learned from a Case of a Traumatic Intracranial Aneurysm, http://www.medschool.lsuhsc.edu/neurosurgery/nervec enter/ traumatican.html.

4. Raju B S, Purohit A K, Murthy S R, Sundaram C, Sanjay T: Traumatic distal anterior cerebral artery aneurysm in a child: a case report. Neurol India 2001;49:295;

5. Paul S. Larson, Andrew Reisner, Dante J. Morassutti, Bassam Abdulhadi, John E. Harpring: Traumatic Intracranial Aneurysms, Neurosurg Focus. 2000;8(1). 